\title{
Minimization of Average Delay, Routing Load and Packet Loss Rate in AODV Routing Protocol
}

\author{
Hemant Kumar Garg \\ Govt. Women Polytechnic College \\ Jaipur, INDIA
}

\author{
P.C.Gupta \\ Jaipur National University \\ Jaipur, INDIA
}

\begin{abstract}
A mobile ad hoc network (MANET) is characterized by energy-limited nodes, bandwidth-constrained, variablecapacity wireless links and dynamic topology, leading to frequent and unpredictable connectivity changes. Since the number of nodes can be large in the network, finding a route to a destination requires frequent exchange of routing control information among the nodes. In this paper we have implemented AODV protocol for ad hoc network, which optimizes delay routing loss rate in ADOV routing through simulator. The network simulator (NS2) is used to show that AODV protocol can reduce network end-to-end delay, increases packet delivery ratio and balance network overload.
\end{abstract}

\section{Keywords}

AODV; Ad hoc network; MANET; routing load; Simulation.

\section{INTRODUCTION}

Mobile Ad hoc network (MANET) is one that comes together as needed, not necessarily with any support from the existing infrastructure or any other kind of fixed stations. In a MANET, no any infrastructure exists and the network topology may dynamically change in the unpredictable manner since nodes are free to move. Routing in MANET depends on many factors including topology, selection of routers, and location of request initiator, and specific underlying characteristics that could serve as a heuristic in finding the path quickly and efficiently. Numerous MANET routing protocols have been proposed. Routing over Ad hoc networks can be broadly classified as topology-based or position-based approaches. Topology-based routing protocols depend on the information about existing links in the network and utilizes them to carry out the task of packet forwarding [1]. They can be further subdivided as being proactive, reactive, or hybrid protocols. Proactive algorithms employ classical routing strategies such as distance vector or link state routing and any changes in the link connection are updated periodically throughout the network. Proactive protocols have the advantages that a node experience minimal delay whenever a route is needed as a route is immediately obtain from the routing table. However proactive protocol may not always be appropriate in MANET's with high mobility. Reactive protocol employs a lazy approach whereby nodes only discover route to destinations on demand. Hybrid protocols combine local proactive and global reactive routing in order to active a higher level of efficiency and scalability. Position based routing algorithms overcome some of the limitations of topology-based routing by relying on the availability of the additional knowledge. These positionbased protocols require that the physical location information of the nodes to be known. Finally, the sender normally uses a location service to determine the position of the destination nodes and to incorporate it in the packet destination address field. Here, the routing process at the each node is based on the destination's location available in the packet and the location of the forwarding node's neighbours.
In this paper complete work is covered in different sections. In the section 2 descriptions about Literature Survey have been shown. Section 3 describes about the proposed work. Section 4 specifies methodology, which is used to solve proposed problem. Section 5 contains the experiments and results. Conclusion is mentioned in section 6 , while in the last section references are mentioned.

\section{LITERATURE SURVEY}

Different work has been already done in Ad hoc routing protocol. In one work Novel AODV-based backup routing scheme for MANET was developed. The proposed scheme utilizes 2 hop neighbour knowledge to establish backup paths during the route discovery phase and maintain updated knowledge of such links. These backup paths are geographically close to the primary path that can repair disrupted links locally without activating a route rediscovery procedure. Additionally, the proposed scheme selects the shortest path containing the largest number of backup path to provide efficient recovery from route failure and maintain an adequate routing length [2].

In another work an optimized AODV protocol called AODV$\mathrm{OA}$ has been used. It is a scheme that improves the hello mechanism, link local repair mechanism and unique route. According to the result of simulation, AODV-OA reduces the average delay caused by route discovery, increase the packet ratio. Meanwhile, it could perform more steadily than AODV in high speed environment [3].

One more work specifies the performance of session in a MANET under it depends heavily on many factors such as the number of multicast senders, receivers, attackers as well as their positions [4]. One more work specifies the Table-driven ad-hoc routing approach DSDV and Ad-hoc on demand routing approach relies on distance or no of hop. The packet delivery of AODV is almost independent of the number of sources. AODV suffers from end to end delays [5].

In this work we have used a network simulator NS2 whose result confirm an intuitive claims: the more attackers there are in the network, more damaging they inflict in to a multicast session in terms of packet delivery ratio, hence it's better to remove such nodes from the network altogether.

\section{PROPOSED WORK}

Our objective in this work is to include exchanging the route information; finding a feasible path to a destination based on criteria such as hop length, minimum power required and life time of the wireless link; gathering information about the path breaks; sending the broken paths expending minimum processing power and bandwidth; and utilizing minimum bandwidth. One of the most important properties of Ad hoc wireless networks is the mobility associated with the nodes. The mobility of nodes results in frequent path break, packet collisions, transient loops, state routing information and difficulty in resource reservation. Since the channel is shared by all nodes in the broadcast region, the bandwidth available per wireless link depend on the number of nodes and the traffic they handle. Thus only a fraction of the total bandwidth 
is available for every node [6]. The bit error rate (BER) in a wireless channel is very high (of the order of $10^{-5}$ to $10^{-3}$ ) compared to that in its wired counterparts (of the order of $10^{-12}$ to $\left.10^{-9}\right)$. Routing protocols designed for Ad hoc wireless networks should take this into account. Consideration of the state of the wireless link, signal-to-noise ratio, and path loss for routing in the Ad hoc wireless networks can improve the efficiency of the routing protocol. The load on the wireless channels varies with the number of nodes present in a given geographical region. This makes the contention for the channel high when the number of nodes increases. The high contention for the channel results in a high number of collisions and subsequent wastage of bandwidth. The constraints on resources such as computing power, battery power and buffer storage also limit the capability of the routine protocol. The route acquisition delay for a node that does not have a route to a particular destination node should be as minimal as possible. This delay may vary with the size of the network and the network load.

Our focus in this work is to identify those nodes in the network which causes loss of packets from source to destination.

\section{METHODOLOGY}

Network simulator NS2 has been used in this work to predict the behavior of the network. Various attributes of the network have been modeled environment to access the network under different conditions. In simulators, the computer network is typically modeled with devices, traffic etc. and the performance is analyzed. Typically, users can then customize the simulator to fulfill their specific analysis needs. Compared to the cost and time involved in setting up an entire test bed containing multiple networked computers, routers and data links, network simulators are relatively fast and inexpensive. They allow engineers, researchers to test scenarios that might be particularly difficult or expensive to emulate using real hardware [7].

\section{Figure 1: Network Simulator}

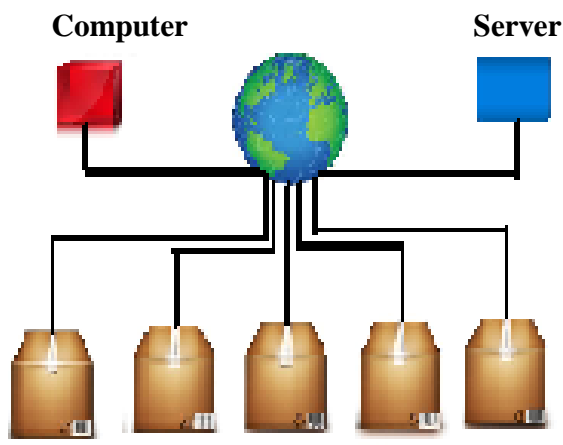

\section{Nodes}

Given below a step wise procedure to achieve our goal:

- Identifying the start and end points in the given network.

- Sending hello messages from source to destination.

- Identifies the list of packet id between nodes in the tabular form.

- If acknowledgement is received back, it means that the route is established between the source and destination node. Otherwise there is no route between them.

- If no route is identified then to find out whether the route broken. Mark the route for under repairing node.

- A mechanism is adopted to repair the broken route.

- If route is broken, then follow an alternative route.

\section{Experiment and Result}

To conduct the experiment a network is considered. This network comprised of 50 nodes placed randomly within $1000 \mathrm{~m} * 1000 \mathrm{~m}$ area. Each node sends out one packet per second with the size of CBR fixed at 512 Bytes. The node moves randomly towards a random spot until it reaches that spot, then it pauses for a while (2s in our simulation) and move again. Different values of pause time and speed (10, 20, 30,40 , and 50) are tested. We use the same environment to simulate the protocol. NS2 simulator has been implemented to evaluate the performance of the used network. Different parameters in the simulation are given in the following table. Table 1: Simulation Parameters

\begin{tabular}{|l|l|}
\hline Routing Protocol & AODV \\
\hline $\begin{array}{l}\text { Number of wireless } \\
\text { nodes }\end{array}$ & 50 nodes \\
\hline Movement space & $1000 * 1000 \mathrm{~m}$ \\
\hline Maximum Speed & $\begin{array}{l}\mathrm{m} / \mathrm{s} \\
\text { Maximum pause time }\end{array}$ \\
\hline Transmission rates & $20,30,40 \& 50$ \\
\hline Packet size & 512 bytes \\
\hline Simulation time & 200 sec \\
\hline
\end{tabular}

\section{Experiment 1:}

Table 2: Route Change with respect to the Pause Time.

\begin{tabular}{|c|c|}
\hline Pause Time & Route Changes \\
\hline 10 & 6304 \\
\hline 20 & 4126 \\
\hline 30 & 4113 \\
\hline 40 & 3408 \\
\hline 50 & 4123 \\
\hline
\end{tabular}

Figure 2: Route Changes

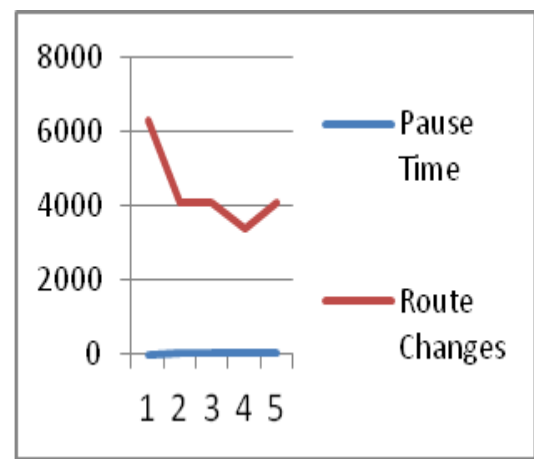


As shown, in the above table and figure, it represents that when we took pause time 10 at a particular node then route changes were 6304 to reach at the destination node. When the pause time is 20 at a particular node then route changes are 4126 , this shows that when we increase the pause time route changes decreases except the pause time 50 .

Table 3: Link Change with respect to the Pause Time.

\begin{tabular}{|c|c|}
\hline Pause Time & Link Changes \\
\hline 10 & 647 \\
\hline 20 & 567 \\
\hline 30 & 454 \\
\hline 40 & 422 \\
\hline 50 & 409 \\
\hline
\end{tabular}

Figure 3: Link Changes

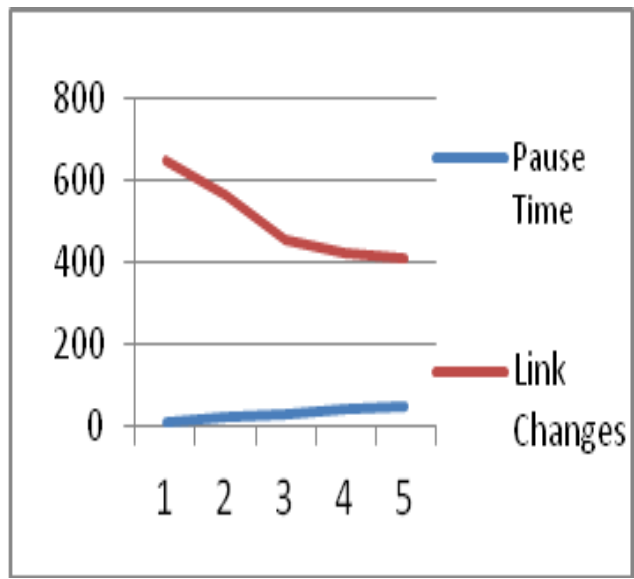

As shown in the table No. 3 and figure 3, it represents that when we took pause time 10 at a particular node then Link changes were 647 to reach at the destination node. When the pause time 20 at a particular node then route changes were 567, this shows that, when we increase the pause time link changes also decreases.

Table 4: Destination Reach with respect to the Pause Time.

\begin{tabular}{|c|c|}
\hline Pause Time & Destination \\
\hline 10 & 49 \\
\hline 20 & 0 \\
\hline 30 & 49 \\
\hline 40 & 49 \\
\hline 50 & 49 \\
\hline
\end{tabular}

Figure 4: Destination Reaches

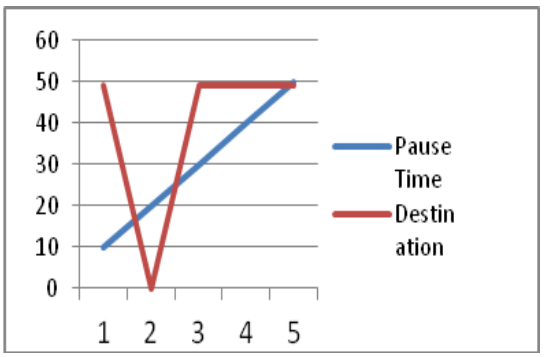

As shown in the table No. 4 and figure 4, it represents that when we took pause time 10 at a particular node, then packet reaches to the destination by travelling 49 nodes, all the pause time behaves similar except pause time 20. At pause time 20 destinations. It represents that at this pause time link of network would have been broken node is zero.

\section{Experiment 2:}

Table 5: Route Change with respect to the Speed.

\begin{tabular}{|c|c|}
\hline Speed & Route Changes \\
\hline 10 & 5437 \\
\hline 20 & 10499 \\
\hline 30 & 10051 \\
\hline 40 & 14407 \\
\hline 50 & 13232 \\
\hline
\end{tabular}

Figure 5: Route Changes

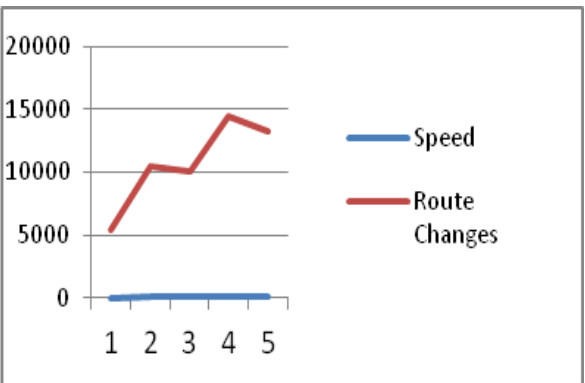

As shown in the table No. 5 and figure 5, it represents that when packet delivery speed is 10 at a particular node then route changes were 5437 to reach at the destination node. When the speed is 20 at a particular node then route changes were 10499, this shows that when we increase the speed of the packet delivery then route changes also increases except the speed 50.

Table 6: Link Change with respect to the Speed.

\begin{tabular}{|c|c|}
\hline Speed & Link Changes \\
\hline 10 & 538 \\
\hline 20 & 921 \\
\hline 30 & 1452 \\
\hline 40 & 1595 \\
\hline 50 & 2012 \\
\hline
\end{tabular}

Figure 6: Link Changes

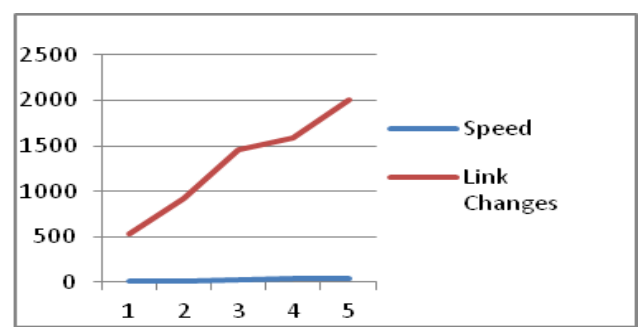

Table 6 and figure 6 , represents that when we took packet delivery speed as 10 at a particular node then Link changes were 538 to reach at the destination node. When the speed is 20 at a particular node then route changes were 921, this 
shows that when we increase the speed then the link changes also increases.

Table 7: Destination Reaches with respect to the Speed.

\begin{tabular}{|c|c|}
\hline Speed & Destination \\
\hline 10 & 0 \\
\hline 20 & 286 \\
\hline 30 & 192 \\
\hline 40 & 187 \\
\hline 50 & 381 \\
\hline
\end{tabular}

Figure 7: Destination Reaches

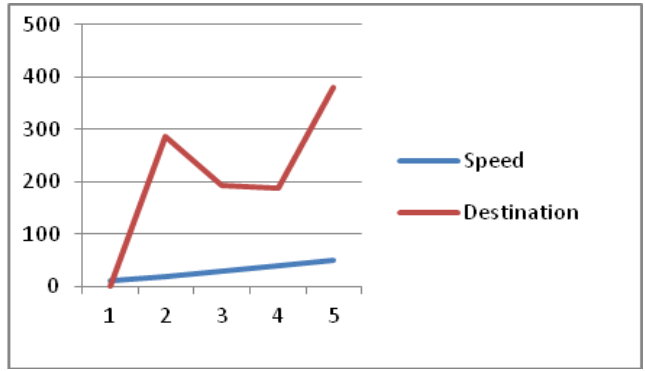

Table No. 7 and figure 7 , represents that when we took packet delivery speed as 20 at a particular node, then packet reaches at destination by travelling 286 nodes, When the speed is 30 at a particular node then destination reaches were 192, this shows that when packet transmission speed increases then the number of nodes decreases to reach at the destination except when the speed is 50. At speed 10 total number of nodes through which packet travels and reaches at destination is zero. Which means link was broken at that moment.

\section{CONCLUSION}

This paper discusses how routing load and packet loss in AODV protocol can be minimized in any given network. Broken link between any two nodes can also be identified and can be repaired or route can be discarded from the network to avoid loss of packets.

\section{ACKLODGEMENT}

We thank all people in the reference. In addition, authors would like to give special thanks to Mrs. Anuradha Garg, Research Scholar, JaganNath University, Jaipur and Mr. R.C.Poonia, Deptt. of Computer Science, Jaipur National University, Jaipur, who gave valuable support for this research work.

\section{REFERENCES}

[1] Ad hoc \& Sensor Networks by Carlos de Morais Cordeiro \& Dharma Prakash Agarwal. Edition 2007.

[2] S.R.Birdar, Koushik Majumder, Subir Kumar Sarkar, Puttamadappa C, Performance Evaluation and Comparison of AODV and AOMDV, IJCSE, Vol.02, No.02, 2010, 373377.

[3] Fei Jiang, \& Jian Jun Hao, Beijing University of Posts and Telecommunications, Beijing, China, IEEE, 2010.

[4] Deepshikha Juneja, "A Novel Approach To Enhance AODV Security In Case of Multicast”, IJMAN, Aug., 2011.

[5] Harish Saini,\& Saba Khanum "QOS Parameter analysis On
AODV Protocolos for Manets" IJMAN, May, 2011.

[6] ADHOC WIRELESS NETWORKS-Architecture and Protocols By C.Siva Ram Murthy \& B.S.Manoj, Ninth Impression, 2011, Pearson.

[7] From Wikipedia, the free encyclopedia. Website:http://en.wikipedia.org/wiki/ Network_ simulation.

[8] C. Perkins, "Ad Hoc On-Demand Distance Vector (AODV) Routing," RFC3561, IETF MANET Working Group, July 2003.

[9] Royer E M, Toh C K, "A Review of Current Routing Protocol for Ad Hoc Mobile Wireless Networks," IEEE Pers Commun Mag, vol. 6, pp. 46-55, Feb. 1999.

[10] Lee S J, Merla G, "AODV-BR: Backup Routing in Ad Hoc Networks," Wireless Communications and Networking Conference, 2000. WCNC. 2000 IEEE, vol. 3, pp. 13111316, Sept. 2000.

[11] E. Royor, Charles E. Perkins, "Evolution and Future Directions of the Ad Hoc On-Demand Distance- Vector Routing Protocol," Ad Hoc Networks Journal, vol. 1,pp. 125150, July 2003.

[12] S. Krco, M. Dupcinov, "Improved Neighbor Detection Algorithm for AODV Routing Protocol," IEEE Communication Letters, vol. 7, pp.584-586, Dec.2003.

[13] Chen Linxing, Zeng Xi, Cao Yi, "Mobile Ad Hoc NetworkSelf Organization Wireless Network Technique,” April 2006

[14] Wang Hongpeng, Lin Cui, "An Enhanced AODV for Mobile Ad Hoc Network," Machine Learning and Cybernetics, International Conference IEEE, vol. 2, pp. 1135-1140, July 2008.

[15] Ge Wenying, Lin Pengwei, "An Optimized AODV Protocol for Ad Hoc Network," Wireless Communications, Network and Mobile Computing Conference IEEE, vol. 2, pp. 1-4, Oct. 2008

[16] Li Yuanzhou," Optimization Strategy for Mobile Ad Hoc Network Based on AODV Routing Protocol”, 2010 IEEE.

[17] N.Venkatadri, K.Ramesh Reddy, Dept. of Computer Science, Vikrama Simhapuri University, Nellore, AP, India. IJMAN, Aug., 2011.

[18] Performance Evaluation of AODV Routing Protocol: RealLife Measurements, Alexander Zurkinden, SCC, June, 2003. 